\title{
Laparoscopic greater curvature plication for treatment for morbidly obese patients: Early experience of Alexandria University
}

\author{
Mohamed A Sharaan, MD; Khaled Katri, MD; Mohamed Hany, MD; \\ Waleed Abdel-Haleem, MD; Tamer AbdelBaki, MD
}

Department of General Surgery, Alexandria University, Alexandria ,Egypt.

\author{
Correspondence: \\ Mohamed Abdallah Sharaan, Department of General Surgery, Faculty of \\ Medicine, Alexandria University, Egypt.
}

\begin{abstract}
Background: Gastric restrictive procedures currently performed for morbidly obese patients include either gastric resection by Laparoscopic Sleeve Gastrectomy or by implanted device (Laparoscopic Adjustable Gastric Banding). We present our early experience of a feasibility study using Laparoscopic Gastric Greater Curvature Plication (LGGCP) for weight loss instead of stapling or banding.

Methods: After approval of the Ethical Committee of Alexandria University Hospital, and taking an informed consent from our patients, we performed LGGCP in 68 patients. After mobilization of the greater curvature, it was folded inwards by two layers of non-absorbable sutures.

Results: Our average Body Mass Index (BMI) was $42 \mathrm{~kg} / \mathrm{m}^{2}$, mean operative time 111 minutes. There was no conversion to laparotomy. The mean percentage of excess weight loss (EWL) at 10 days, 1 month, 3 months, 6 months and 12 months were 10\%, 16\%, 25\%, 35\%, and $56 \%$ respectively. No intra-operative complications, no mortality, $5.8 \%$ major complications in the form of one case of fundic herniation that required laparoscopic partial gastrectomy, and 3 cases of obstructed pouch, one required undo-plication, the other one was relieved by medical treatment, and the last one improved by upper endoscopic dilatation.

Conclusion: Our early experience has suggested that LGGCP is a successful way to reduce the stomach capacity, and an acceptable early weight loss. LGGCP is safe and feasible but it is not without complications. Prospective randomized study between LGGCP and Laparoscopic Sleeve Gastrectomy is needed.
\end{abstract}

\section{Introduction:}

Morbid obesity is a well-recognized growing health problem worldwide. ${ }^{1}$ Diet, exercise, and or medication as a treatment have not demonstrated sustainable clinically significant results. ${ }^{2}$ Bariatric surgery has a positive impact as a primary therapy for the treatment of obesity and its comorbidities. However, there is a significant debate concerning which patients are optimal candidates for which procedures. ${ }^{3,4}$

Many types of restrictive procedures have been performed to achieve weight loss. ${ }^{5}$ Most of these procedures have been abandoned because of poor long-term weight loss, food intolerance, or severe gastro-esophageal reflux and complications. The main aim of these procedures was to design the stomach 
into proximal stomach horizontally or vertically with a small outlet. Vertical banded gastroplasty, in particular, has resulted in poor long-term outcomes, and a high percentage of vertical banded gastroplasty patients have required revision to Roux-en-Y gastric bypass to alleviate intolerable reflux symptoms and dysphagia or to achieve weight loss again. ${ }^{6-12}$ Laparoscopic adjustable gastric banding and sleeve gastrectomy are currently the wellknown gastric restrictive procedures. The risk of gastric slippage, band erosion associated with the gastric band, and the risk of gastric leakage with sleeve gastrectomy has limited the acceptance of these procedures by some patients and surgeons. Recently, endo-luminal procedures have been developed to achieve a similar restrictive effect without subjecting the patient to the risk of surgery. ${ }^{13-14}$ However, these endoscopic procedures were not durable because they entail approximation of mucosa of opposing gastric walls. ${ }^{14}$ The aim of the present study was to assess the feasibility, safety and early outcome laparoscopic greater curvature plication (LGCP), which is a new restrictive bariatric surgical technique that creates gastric restriction without the use of an implant and without performing gastric resection and so eliminates the complications associated with gastric band and Sleeve Gastrectomy.

\section{Methods:}

From April 2011 to April 2012, we prospectively performed Laparoscopic Gastric Greater Curvature Plication (LGGCP) to 68 obese patients. This procedure was approved by the ethical committee of the hospital, and all patients signed an informed consent before going to surgery and received a pre-operative prophylactic dose of low molecular weight heparin. Inclusion criteria were BMI above $35 \mathrm{~kg} / \mathrm{m}^{2}$, age from 18 to 65 years, patients with no previous gastric surgeries.

The exclusion criteria included pregnancy or lactation at screening or surgery, a documented history of drug and/or alcohol abuse within 2 years of the screening visit, previous malabsorptive or restrictive procedures performed for the treatment of obesity, the participation in any other investigational device or drug study within 12 weeks of enrollment, severe cardiopulmonary disease or other serious organic disease, uncontrolled hypertension, and portal hypertension, and patients on hormonal therapy.

\section{Surgical procedure:}

All our patients were subjected to general anesthesia, and positioned supine and legs opened wrapped by elastic bandage or elastic stocking. Trocar placement was as follows: one $10-\mathrm{mm}$ trocar above and slightly to the right of the umbilicus for the $30^{\circ}$ laparoscope; one $10-\mathrm{mm}$ trocar in the upper right quadrant (URQ) for passing the needle, for suturing, and for the surgeon's right hand; one 5-mm trocar also in the URQ below the $10-\mathrm{mm}$ trocar at the axillary line for the surgeon's assistant; one 5-mm trocar below the xiphoid process for liver retraction; and one $5-\mathrm{mm}$ trocar in the upper left quadrant (ULQ) for the surgeon's left hand. The procedure began with the dissection of the Greater Curvature of the stomach $6 \mathrm{~cm}$ proximal to the pylorus extending until reaching the Angle of His and the removal of the fat pad in this location, using the Harmonic ${ }^{\mathrm{TM}}$ scalpel (Ethicon EndoSurgery, Inc., Cincinnati, Ohio). Once the access to the posterior wall was achieved. Occasionally, posterior gastric adhesions were also dissected to allow optimal freedom of the stomach to facilitate creation of the invagination easily. Then we allowed passage of a calibrating tube 32-Fr Bougie, to be placed near the lesser curvature , then initiate gastric plication by taking non-absorbable sutures invaginating the greater curvature over the tube, starting $1-2 \mathrm{~cm}$ from the angle of His, with fixation of the fundus by the first suture. Then continuing the application of the first row of extra-mucosal interrupted stitches of 2-0 Ethibond ${ }^{\mathrm{TM}}$ (Ethicon, Inc., Somerville, NJ, USA) sutures. The distance between each suture of the first row was at least $2 \mathrm{~cm}$. This row was followed by a second subsequent row created with extra-mucosal running suture of 2-0 Prolene ${ }^{\mathrm{TM}}$ (Ethicon, Inc., Somerville, NJ, USA). Leak tests were performed 
with methylene blue in all cases. Upper GI endoscopy was routinely performed in all of our cases to assess the final stomach capacity and to confirm the patency of the created gastric pouch. A drain was placed beside the suture line in all of our cases. Postoperatively, patients were followed and monitored for any complications and discharged as soon as they tolerated fluid diet and received a daily proton-pump inhibitor, for 6 weeks, anti-emetic (Ondasentron) and the antispasmodic (Hyoscine) were prescribed for 7 days. The postoperative diet of fluids for 2 weeks, then soft diet for another 2 weeks, and starting semi-solid and solid diet in a stepwise fashion, while restricting sweet diet. We followed the patients 1 week and then at $1,3,6,12 \& 18$ months in the postoperative period. Gastrograffine study of the created gastric pouch was scheduled once 12 months postoperative.

\section{Results:}

Our study included a total of 68 patients, 18 males (26.4 \%), 50 females (73.6\%). Their mean age was 35 years (range 18-65 years), their mean weight was $116 \mathrm{~kg}$ (range 80$192 \mathrm{~kg}$ ) and the average preoperative BMI was $42 \mathrm{~kg} / \mathrm{m}^{2}$ (range 32.5-67.1). The mean operative time was 111 minutes (range 70180 minutes). The mean hospital length of stay was 25.92 hours. All procedures were performed laparoscopically without convergence to laparotomy. On average, patients returned to normal activities 5 days (4 to 13 days) following surgery. Mean total weight loss (TWL) was calculated to be $7 \%$ TWL at 3 months (5 patients), 10\% TWL at 6 months (7 patients), $14.7 \%$ TWL at 12 months (10 patients) postoperatively respectively. Mean percentage of excess weight loss (\% EWL) was calculated to be $10 \%$ EWL at 10 days, $16 \%$ EWL at 1 month, 25\% EWL at 3 months, 35\% EWL at 6 months, 56\% EWL at 12 months postoperatively respectively. No intra-operative complications were documented. In the postoperative period we recorded $55 \%$ of cases had recurrent vomiting in the first week postoperatively. These cases were responding well to medical treatment. About $84 \%$ of cases complained of recurrent colicky abdominal pain which was exaggerated in response to fluids, this was in the first postoperative week, it was relieved by fixed dose of anti-spasmodics for about 1 week. We had three cases of obstructed pouch, which were all presented by recurrent vomiting including their saliva, abdominal pain, and severe colicks, and it was confirmed by gastrograffine study denoting failure or difficult passage of the dye through the pouch. The first case was narrowed inlet of the pouch by infolding of the gastric fundus obstructing the osophago-gastric junction, this case was mild degree and was improved by medical treatment, while the second case was the same as the first one but in moderate degree and was relieved by endoscopic dilatation of the inlet of the pouch. The third case of obstructed pouch was at the outlet, was discovered also by gastrograffine study, was not improved neither by medical treatment nor by endoscopic dilatation for 4 weeks, so a decision for laparoscopic undo-plication was taken and we removed all the stitches at the outlet of the pouch, and the case improved well after the procedure without leakage which was confirmed also by gastrograffine study. We had a case of fundus herniation between the first stitch and the osophagogastric junction, this case was persistently complaining of left shoulder pain and colicks not improving by medications without vomiting, fever or even intra-abdominal collection, we discovered it by gastrograffine study and CT scan, so a decision was made to re-operate this case laparoscopically, we found a big sized herniated stomach elevating and adherent to the diaphragm and the spleen, it was viable stomach but hugely distended , so we removed stitches in the neck of herniated stomach and in an attempt to dissect its adherence with the spleen and diaphragm we discovered that it was perforated and sealed with its adherence with diaphragm, so we aspirated its contents and we did a partial gastrectomy to it by stapler (Echlon $60 \mathrm{~mm}$ gold fire), we added suture overseweing the staple line. This case passed a smooth postoperative course without any leakage 


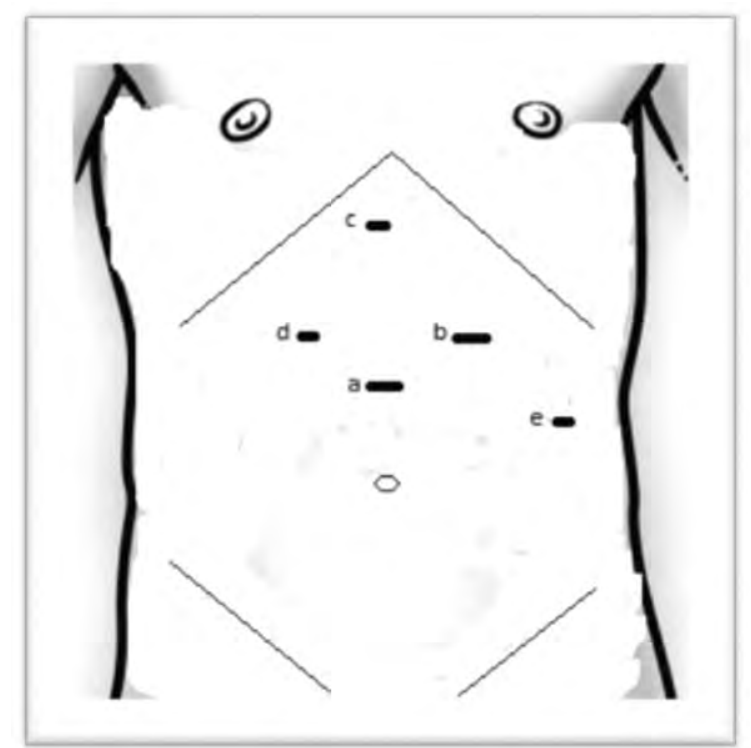

Figure (1): Trocar sites in LGGCP, a. Camera trocar 10mm, b. Right hand trocar and needle insertion trocar $10 \mathrm{~mm}$, c. Liver retractor trocar $5 \mathrm{~mm}$, d. Left hand trocar $5 \mathrm{~mm}$, e. Assistant trocar $5 \mathrm{~mm}$.

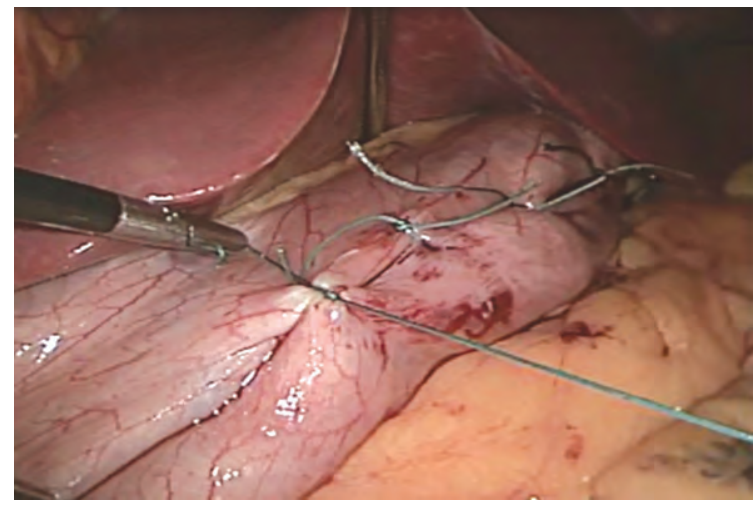

Figure (2): Intra-operative picture of the first rows of interrupted non-absorbable sutures.

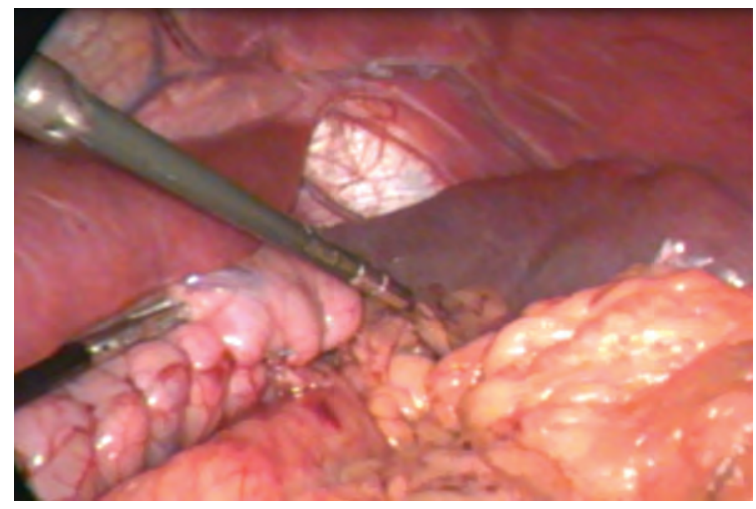

Figure (4): Final intra-operative picture of $L G G C P$.

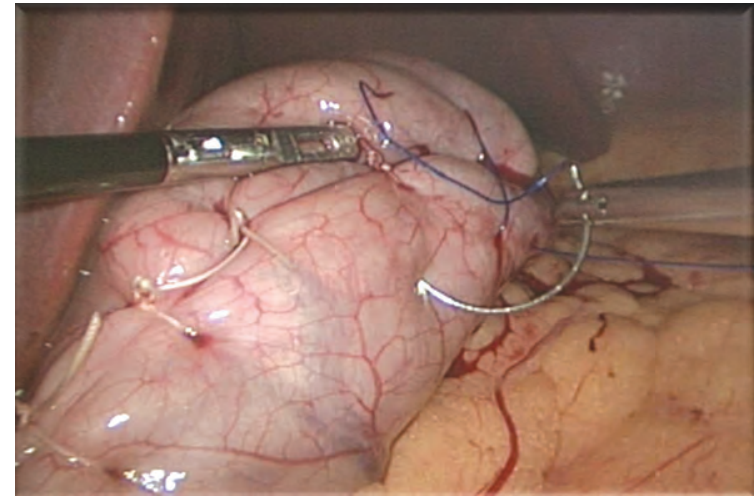

Figure (3): Intra-operative picture of the second row of continuous non-absorbable sutures.

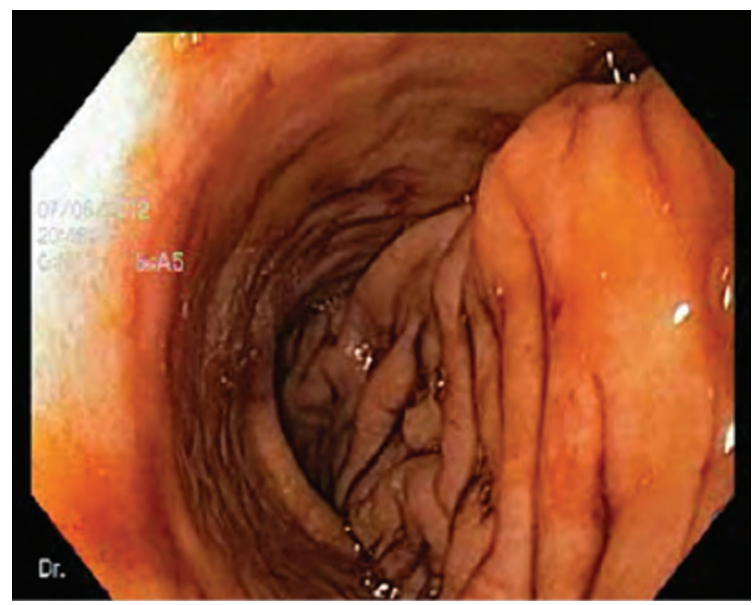

Figure (5): Intra-operative endoscopic view of LGGCP. 


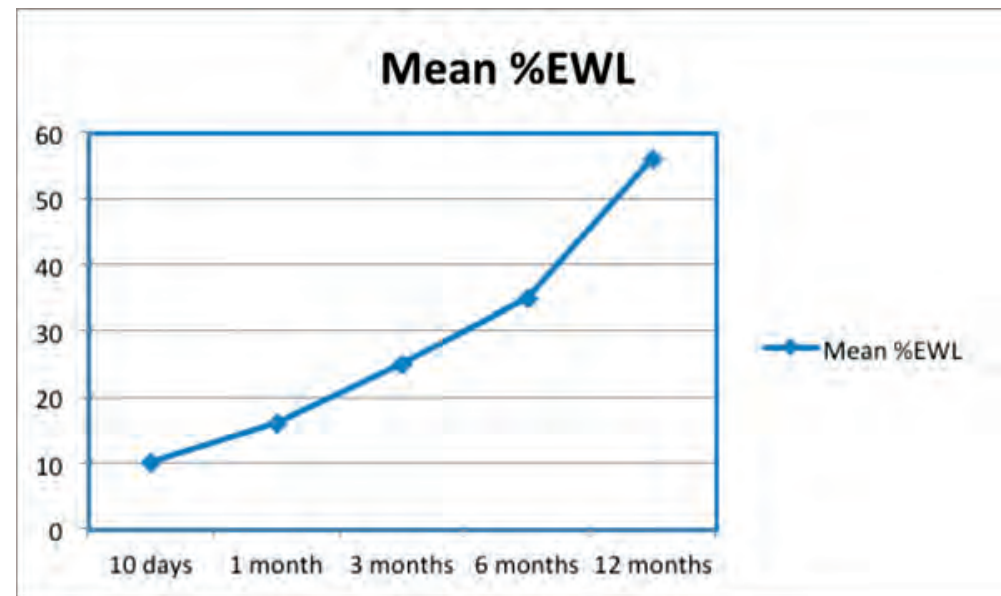

Figure (6): Mean percentage of excess weight loss expressed in \%EWL with the LGGCP procedure at 10 days,1, 3, 6 and 12 months.

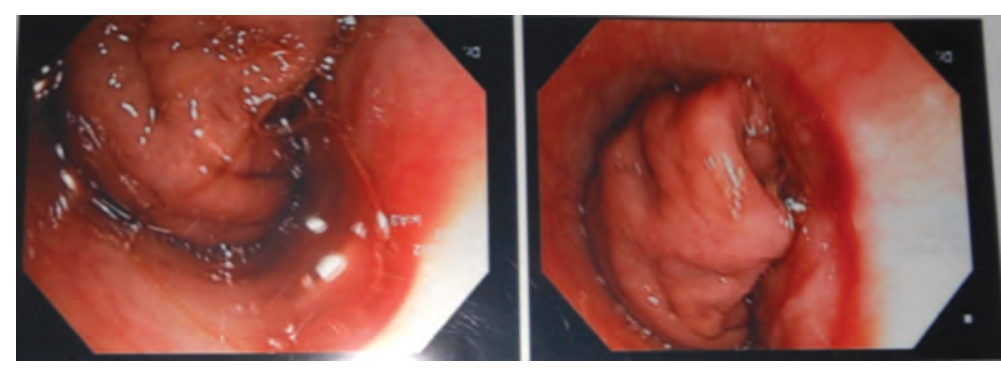

Figure (7): Post-operative endoscopic view of obstruction of the inlet of LGGCP pouch by the gastric fundus.

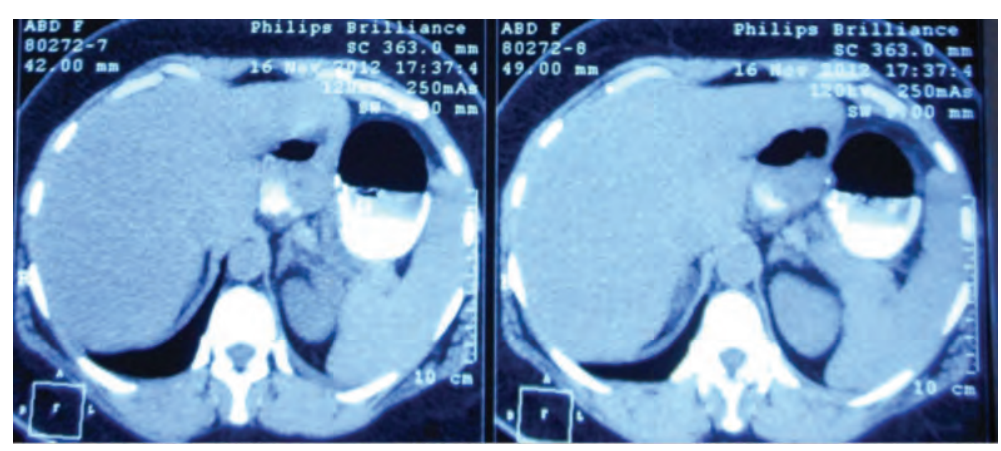

Figure (8): CT abdomen with oral contrast showing big fundus herniation.

Table (1): Comparison between the present study and other studies in LGGCP.

\begin{tabular}{|c|c|c|c|c|c|c|c|c|}
\hline & Patients & Operative time & Mean 8MI & XEWL at IM & SEEWL 3M & KEWL $6 M$ & SEWL12M & Major Complications \\
\hline Ramos et a $\left.\right|^{(a)}$ & 42 & $50 \mathrm{mn}$ & 41 & 20 & 32 & 48 & 60 & no \\
\hline Skeras et a! ${ }^{[33\}}$ & 335 & 58 & 39.5 & $\ldots$ & 34 & 51 & 67 & $8.80 \%$ \\
\hline Talebpour et al ${ }^{1321}$ & 800 & 72 & 42 & 20 & 45 & 60 & 67 & $1.60 \%$ \\
\hline Our Present Study & 68 & 211 & 42 & 16 & 25 & 35 & 56 & $5.8 \%$ \\
\hline
\end{tabular}

confirmed by gastrograffine study. There has been no record of weight regain in any patient to date.

\section{Discussion:}

Bariatric procedures reducing stomach capacity to promote mechanical restriction to food intake is one of the traditionally accepted mechanisms used to promote weight loss. There are at least two surgical procedures that appear to rely on this principle, Adjustable Gastric Band (AGB), and Vertical Sleeve Gastrectomy (VSG). ${ }^{16}$ AGB has been used for many years and offers surgical ease, adjustability, reversibility as well as low immediate mortality and morbidity rates. ${ }^{18}$ In 
terms of weight loss, AGB achieves around $50 \%$ EWL, but unsatisfactory weight loss occurs in more than $20 \%$ of patients. 15,19 AGB also has the disadvantage of requiring a long-term implant, which has been shown to dislodge and/or erode in the stomach in up to $11 \%$ of patients. ${ }^{20}$ This suggests a failure rate requiring surgical revision in up to $25 \%$ of patients. ${ }^{25}$ These secondary procedures can be challenging and difficult. ${ }^{21}$ VSG is a procedure initially used as the first stage of a definitive bariatric treatment known as the duodenal switch.22 Vertical gastrectomy of the greater curvature is performed, resulting in a tubular stomach with the purpose of restricting food intake. As a primary bariatric procedure, medium-term results have been shown to be adequate (greater than 60\% EWL), with improvements in co-morbidities such as type 2 diabetes, hypertension, and obstructive sleep apnea in more than $65 \%$ of cases. $^{23}$ These promising results are associated with some complications, however, such as esophagites, stenosis, fistulas, and gastric leaks near the angle of His. These leaks and fistulas are reported in nearly $1 \%$ of cases and can be very difficult to treat. ${ }^{17,22,24}$ The bariatric procedure that offer restriction of gastric capacity without adding a permanent implant also without resection and stapling of the stomach with possibility of leaks, are highly desirable. Laparoscopic Greater Curvature Plication (LGGCP) is a relatively new technique. It was initially proposed by Wilkinson and Peloso 27 in 1981 and introduced in 2006 by Dr Talebpour in Iran. ${ }^{26}$ It is similar to Laparoscopic Sleeve Gastrectomy (LSG) as they both resulted in gastric tube formation and elimination of the greater curvature. However, LGGCP spared gastric resection and the use of an implant. The advantages of LGGCP that mostly influenced the patients' decision-making were the minimal invasiveness of the operation, the lack of gastric resection, the absence of foreign bodies, and the convenient follow-up.

In two separate papers, Fusco et al, report efficacy in gastric plication procedures, as measured by changes in the weight progression of rats. ${ }^{28,29}$ In one paper, Fusco et al, reported an increased effect from plication of the greater curvature when compared to plication of the anterior surface. These results are in agreement with initial clinical reports by Brethauer et al, who reported an increased weight loss in patients receiving LGGCP when compared to plication of the anterior surface. ${ }^{30}$ In the present study, there were no convergence to laparotomy and the mean operative time was 111 minutes, mean hospital stay 25 hours, mean excess weight loss was $25 \%$ at 3 months, $35 \%$ at 6 months, and $56 \%$ at 12 months. Ramos et al., in their series of 42 cases, ${ }^{4}$ reported a mean operative time of 50 minutes (40-100 minutes) and a mean hospital stay of 36 hours (24 to 96). Mean TWL on 1, 3, 6, 12, and 18 months from the operation was $10 \%, 15 \%, 22 \%, 28 \%$, and $30 \%$, respectively, with mean \% EWL 20\% for the 1 st month, $32 \%$ at 3months, $48 \%$ at 6 months, $60 \%$ at 12 months, and $62 \%$ at 18 months. Only minor complications were observed, with symptoms such as nausea vomiting and sialorrhea up to $35 \%$ resolving spontaneously within 2 weeks. ${ }^{4}$

In the present study, we reported three cases of obstructed pouch, in two of them the cause was due to fundic mucosa obstructing the osophago-gastric pouch, one of them improved by medical treatment the other one improved by upper endoscopy and dilatation, while in the third case the obstruction was at the incisura angularis, and did not improve by any means, so reoperation and undo-plication of the two layers of the last $4 \mathrm{~cm}$ of the pouch was done. This was under endoscopic guidance and the case passed a smooth postoperative course without any leak. Brethauer et $\mathrm{al}^{31}$ reported one case of obstruction of the gastric lumen by the intraluminal fold after LGGCP, this was in the area of the incisura and they claim that this area is particularly at risk of this complication if the intraluminal fold infringes on the lesser curvature or creates a kink in the lumen. They also claimed that this problem can be managed conservatively until edema of the mucosa subsided, but the patient had intolerablilty to fluids so they reoperated on him by removing the outer row of sutures, and replicating the outer row more 
loosely to restore some of the gastric lumen. This patient did well after the reoperation, and had excellent weight loss. ${ }^{31}$

Talebpour et al, 32 reported in their large study including 800 cases, three cases of pouch obstruction due to displacement of released fundus outside the suture line and extra-expansion the displaced folds stretched the string, tightening the rest of the knots especially the last one near pylorus. The stomach outflow kinked and produced an obstruction. The management was via laparoscopy. The suture line was undone and replication performed. The last tie close to the pylorus was done relatively looser than before. We believe that an important step in the LGGCP procedure is loose suture near the incisura angularis, and an intra-operative upper endoscopy to avoid risk of pouch obstruction.

In the present study, we had a case of fundus herniation outside the stuture line, with sealed perforation that required reoperation by laparoscopy and resection of the herniated stomach with over-running suture. This patient passed a smooth post-operative course and had an excellent weight loss.

Also in their large series of LGGCP, Talebpour et al, ${ }^{32}$ had postoperative technical complications in 8 cases out of 800 (1\%). Micro perforation occurred in three cases; the first one occurred at the site of gastric holding by grasper at pre-pyloric area which was closed by simple suture without any change in plication via laparotomy; one case at the site of needle insertion at upper end of plication due to increased intraluminal pressure and its dilation in one point which was treated by simple suture by laparoscopy; and the last one due to fundus sliding outside of suture row and blowout of dilated displaced fundus. Treatment of this case was by laparotomy, undoing the suture line and drain insertion. During follow up it took about 2 weeks for fistula to evolve and closure of fistula was completed after 45 days and drains were taken out afterwards.

In our present study we had no mortality, no weight regain, $5.8 \%$ major complication (4 out of 68 cases) in the form of obstructed pouch and herniated fundus. We modified our technique later on in patients not included in this study, by taking the first row interrupted non absorbable sutures but taking it in four points so that there will be 3 small folds instead of one big fold. This is to avoid obstruction of the pouch in the osophago-gastric junction and at the level of the incisura angularis. Also by this technique the fundus will be fixed and it cannot slide to herniate. We believe that LGGCP is not without complications, taking the benefit of learning for our and other complications is important issue to avoid its occurrence.

\section{Conclusion:}

Our early experience has suggested that LGGCP is a successful way to reduce the stomach capacity, and an acceptable early weight loss. LGGCP is safe and feasible but it is not without complications. We did modifications of the technique of suturing to avoid the related complications. Prospective randomized study between LGGCP and Laparoscopic Sleeve Gastrectomy is needed.

\section{References:}

1- Prentice AM: The emerging epidemic of obesity in developing countries. Int $J$ Epidemiol 2006; 35(1): 93-99.

2- Sjostrom L, Narbro K, Sjostrom CD, et al: Effects of bariatric surgery on mortality in Swedish obese subjects. $N$ Engl J Med 2007; 357(8): 741-752.

3- Buchwald H, Avidor Y, Braunwald E, et al: Bariatric surgery: A systematic review and meta-analysis. JAMA 2004; 292(14): 1724-1737.

4- Ramos A, Galvao Neto M, Galvao M, et al: Laparoscopic greater curvature plication: Initial results of an alternative restrictive bariatric procedure. Obes Surg 2010; 20: 913-918.

5- Buchwald H, Buchwald JN: Evolution of operative procedures for the management of morbid obesity 1950-2000. Obes Surg 2002; 12: 705-717.

6- Balsiger BM, Poggio JL, Mai J, Kelly KA, Sarr MG: Ten and more years after vertical banded gastroplasty as primary operation 
for morbid obesity. J Gastrointest Surg 2000; 4: 598-605.

7- Ramsey-Stewart G: Vertical banded gastroplasty for morbid obesity: Weight loss at short and long-term follow up. Aust N Z J Surg 1995; 65: 4-7.

8- Salmon PA: Failure of gastroplasty pouch and stoma size to correlate with postoperative weight loss. Can J Surg 1986; 29: 60-63.

9- MacLean LD, Rhode BM, Forse RA: Late results of vertical banded gastroplasty for morbid and super obesity. Surgery 1990; 107: 20-27.

10-Kim CH, Sarr MG: Severe reflux esophagitis after vertical banded gastroplasty for treatment of morbid obesity. Mayo Clin Proc 1992; 67: 33-35.

11-Nightengale ML, Sarr MG, Kelly KA, Jensen MD, Zinsmeister AR, Palumbo PJ: Prospective evaluation of vertical banded gastroplasty as the primary operation for morbid obesity. Mayo Clin Proc 1991; 66: 773-782.

12-Balsiger BM, Murr MM, Mai J, Sarr MG: Gastroesophageal reflux after intact vertical banded gastroplasty: Correction by conversion to Roux-en- $\mathrm{Y}$ gastric bypass. J Gastrointest Surg 2000; 4: 276-281.

13-Moreno C, Closset J, Dugardeyn S, et al: Transoral gastroplasty is safe, feasible, and induces significant weight loss in morbidly obese patients: Results of the second human pilot study. Endoscopy 2008; 40: 406-413.

14-Deviere J, Ojeda Valdes G, Cuevas Herrera L, et al: Safety, feasibility and weight loss after transoral gastroplasty: First human multicenter study. Surg Endosc 2008; 22: 589-598.

15-Buchwald H, Avidor Y, Braunwald E, et al: Bariatric surgery: A systematic review and meta-analysis. JAMA 2004; 292 (14): 1724-1737.

16-DeMaria EJ: Bariatric surgery for morbid obesity. $N$ Engl J Med 2007; 356(21): 2176-2183.

17-Baltasar A, Bou R, Bengochea M, et al: Use of a Roux limb to correct esophagogastric junction fistulas after sleeve gastrectomy. Obes Surg 2007; 17(10): 1408-1410.

18-O’Brien PE: Laparoscopic adjustable gastric banding: A real option for a real problem. ANZ J Surg 2003; 73(8): 562.

19-Martin LF, Smits GJ, Greenstein RJ: Treating morbid obesity with laparoscopic adjustable gastric banding. Am J Surg 2007; 194 (3): 333-343.

20-Campos J, Ramos A, Galvao NM, et al: Hypovolemic shock due to intragastric migration of an adjustable gastric band. Obes Surg 2007; 17(4): 562-564.

21-Tice JA, Karliner L, Walsh J, et al: Gastric banding or bypass? A systematic review comparing the two most popular bariatric procedures. Am J Med 2008; 121(10): 885-893.

22-Gumbs AA, Gagner M, Dakin G, et al: Sleeve gastrectomy for morbid obesity. Obes Surg 2007; 17(7): 962-969.

23-Silecchia G, Boru C, Pecchia A, et al: Effectiveness of laparoscopic sleeve gastrectomy (first stage of biliopancreatic diversion with duodenal switch) on co-morbidities in super-obese highrisk patients. Obes Surg 2006; 16(9): 1138-1144.

24-Campos JM, Siqueira LT, Meira MR, et al: Gastrobronchial fistula as a rare complication of gastroplasty for obesity: A report of two cases. J Bras Pneumol 2007; 33(4): 475-479.

25-Toouli J, Kow L, Ramos AC, et al: International multicenter study of safety and effectiveness of Swedish adjustable gastric band in 1-, 3-, and 5-year followup cohorts. Surg Obes Relat Dis 2009; 5: 598-609.

26-Wilkinson L, Peloso O: Gastric (reservoir) reduction for morbid obesity. Archives of Surgery 1981; 116(5): 602-605.

27-Talebpour M, Amoli B: Laparoscopic total gastric vertical plication in morbid obesity. Journal of Laparoendoscopic and Advanced Surgical Techniques 2007; 17(6): 793-798.

28-Fusco PE, Poggetti RS, Younes RN, et al: Evaluation of gastric greater curvature invagination for weight loss in rats. Obes 
Surg 2006; 16(2): 172-177.

29-Fusco PE, Poggetti RS, Younes RN, et al: Comparison of anterior gastric wall and greater gastric curvature invaginations for weight loss in rats. Obes Surg 2007; 17(10): 1340-1345.

30-Brethauer SA, Harris JL, Chand B, Kroh M, Rogula T, Schauer PR: Initial results of vertical gastric plication for severe obesity. Society of American Gastrointestinal and Endoscopic Surgeons. Phoenix, Arizona. April 22-25, 2009.

31-Brethauer S, Harris J, Kroh M, et al: Laparoscopic gastric plication for treatment of severe obesity. Surg Obes

Relat Dis 2011; 7(1): 15-22.

32-Talebpour M, Motamedi SM, Talebpour $A$, et al: Twelve year experience of laparoscopic gastric plication in morbid obesity: Development of the technique and patient outcomes. Ann Surg Innov Res 2012; 6(1): 1164-1166.

33-Skrekas G, Antiochos K, Stafyla VK: Laparoscopic gastric greater curvature plication: Results and complications in a series of 135 patients. Obes Surg 2011; 21(11): 1657-1663. 